From the numerous examinations of soil samples taken, certain areas where salt and alkalinity are high were indicated as being fit, as yet, only for rice crops. Others proved to be of better quality, and it is held that the failure to appreciate this is due to lack of knowledge of the proper method of rotation, the essential feature of which is to introduce a leguminous crop immediately after the rice in order to restore the nitrogen balance. The anrerobic soil conditions under which rice is grown have the result of reducing the nitrogen available for the succeeding crop. A study of the natural flora of the area showed that these gave reliable indications as to the state of the land.

This forms an interesting commentary on the third of these researches, which was suggested by the dominance of particular types of natural flora noticed in certain localities surveyed in 1931-32. The purpose of this investigation was to determine the class of soil fuvoured by each of the five types of vegetation selected, as a guide in future soil surveys. In "A Study of the Soil Profiles of the Punjab Plains with Reference to their Natural Flora" R. C. Hoon and M. L. Mehta deal fully with each case, and, in the result, show that four of the botanical types referred to-Prosopis spicigera, Salvadora oleoides, Tamarix articulata and Capparis aphylla-are re. liable indicators of the soils they favour, and deductions may be drawn as to the crops for which they are suitable and the precautions necessary when under irrigation and treatment. In conjunction with grass (Eleusine agyptica), however, a wide range of soils was found and from this type of vegetation, therefore, no index value was obtainable.

\title{
The Aliphatic Diazo Compounds
}

$I^{N}$ the forty-second Bedson Club Lecture delivered in King's College, Newcastle-upon-Tyne, Prof. J. Kenner discussed the aliphatic diazo compounds. The observation that the naturally occurring amide asparagine lost nitrogen when nitrous acid was present in nitric acid used for its hydrolysis led to the discovery of the general effect of this substance upon aliphatic amines. The work of Griess on aromatic diazo compounds was followed by the discovery of diazo acetic ester by Curtius, who turned to good account the suggestion of Baeyer that the acid part of glycine should be esterified whilst investigation of the amino group was proceeding. Together with Buchner, Curtius described the properties of the new substance which are responsible for the importance of its congeners to-day.

With regard to the formula of the aliphatic diazo compounds Curtius favoured the cyclic structure,

$$
\text { R.CH }
$$

but Thiele was led by observations of Staudinger and himself to propose $\mathrm{R} . \mathrm{CH}=\mathbf{N} \equiv \mathbf{N}$. Modern theory regards these compounds as examples of mesomerism, and the suggestion was now put for. ward that their formation from the diazo-hydroxides first produced, for example, in the preparation of diazomethane from nitrosomethylurethane, is due to the peculiar stability of the mesomeric state.

Prof. Kenner's active interest in the subject arose from his investigations of the tendency of the nitroso-group to pass whenever possible into the oximino-group. This led to an improvement of the early von Pechmann preparation of diazo paraffins, consisting in brief in the alkaline decomposition of the $\mathrm{N}$-nitroso derivative of the addition product of mesityl oxide and a primary aliphatic amine.

On the larger scale, certain difficulties were encountered. Loss was occasioned by the liberated diazoparaffin combining with the mesityl oxide simultaneously formed, but was reduced by working at lower temperatures and by separating mesityl oxide from the distillate by a fractionating column. In the case of higher homologues the boiling points of which approximate to that of mesityl oxide, pulegone can with advantage be substituted for the latter, in the preparation of the nitroso compound. Ether, first used to carry away the diazo compounds, was recognized by Meerwein to inhibit their reactivity, but a very satisfactory technique was developed in which anisole is used as solvent and nitrogen as a carrier gas under reduced pressure.

Vinyldiazomethane and its methyl derivative exhibit a comparatively feeble reactivity towards acids ; this was attributed to internal saturation, and in agreement with this the vinyl compound readily passes by monomolecular reaction into pyrazole.

After a discussion of some of the properties of the aliphatic diazo compounds, their chemical behaviour was discussed and more particularly towards ketones. The inertia of these towards diazomethane was first overcome when Meerwein activated acetone by means of methyl alcohol. The initial attack of the diazo compound at the carbon atom of the carbonyl group is followed by loss of nitrogen and stabilization, either by formation of an ethylene oxide ring or by a Wagner-Meerwein type of rearrangement. This latter, in the particular case of cyclic ketones, results in ring enlargement; cyclo-hexanone, for example, gives on treatment with diazomethane, 46 per cent cyclo-heptanone. Similar reactions have been carried out with the homologues of diazomethane (which are more reactive than the parent substance), and numerous 6-, 7- and 8-membered 2-alkyl cyclo. ketones have thus been prepared. The same products were prepared directly from the corresponding nitrosourethanes by a procedure applied by Meerwein to nitrosomethylurethane, and this has the advan. tage of applicability in cases in which it may not be possible to prepare the diazo compound itself. By this device also it is possible to introduce groups into a ketone which may be of use in further synthetic operations. 